\title{
Comercio de derechos de emisión, adopción de tecnología y heterogeneidad de industrias: un enfoque experimental*
}

\author{
Eva Camacho Cuena \\ Dpto. de Análisis Económico, Universidad Autónoma de Madrid \\ Dpto. de Economía, Universitat Jaume I de Castellón \\ Till Requate \\ Dpto. de Economía, Universidad de Kiel \\ Israel Waichman \\ Dpto. de Economía, Universidad de Kiel \\ José Luis Zofío \\ Dpto. de Análisis Económico, Universidad Autónoma de Madrid
}

\begin{abstract}
Resumen
Los beneficios económicos y ambientales esperados de una determinada política medioambiental basada en el comercio de derechos de emisisón dependen de cómo éstos son implementados en el campo. En este artículo, analizamos el comercio de derechos de emisión a través de un mecanismo de subasta doble combinado con una estructura de la industria que presenta heterogeneidad en los costes de reducción de emisiones de las empresas, donde se permite además la adopción de una nueva tecnología más limpia. Nuestros resultados muestran que existe una relación directa entre la heterogeneidad de la tecnología dentro de una industria, la eficiencia del comportamiento inversor de las empresas, así como la eficiencia del mecanismo de mercado para extraer los beneficios potenciales derivados de una reasignación de los permisos de emisión.
\end{abstract}

Clasificación JEL: C92; D44; L51; Q28; Q55.

Palabras clave: política medioambiental, tecnologías de mitigación, permisos de emisión, heterogeneidad de empresas, experimento.

\section{Abstract}

The economic and environmental gains expected from particular environmental policies based on allowances trading programs depend on how they are specifically implemented in the field. In this paper we focus on a tradable permit program based on a double auction market combined with an industry structure that presents heterogeneity in conventional abatement cost functions across firms, and allows for the possibility of adopting a new low emissions technology. Our results show that there is a direct relationship between the technological heterogeneity within an industry and the efficiency

* Agradecemos el apoyo financiero de Fritz Thyssen Stiftung (AZ 10.07.1.124), de la Universidad Autónoma de Madrid y de las Becas de Investigación de la Comunidad de Madrid (009/SHD/05 y CCG07-UAM-HUM-1963). Traducción a cargo de Jose Manuel Puerta. 
of firms behavior when investing in cleaner technologies and the efficiency of the market when reaping the potential benefits from trade by way of permits reallocation.

JEL classification: C92; D44; L51; Q28; Q55.

Keywords: environmental policy, abatement technology, emission permits, firm heterogeneity, experiment.

\section{Introducción}

La teoría y práctica de la política medioambiental se ha convertido en uno de los temas de mayor interés en las últimas décadas. En este campo, es ampliamente reconocido por los académicos que los instrumentos de control de emisiones que incentiva la reducción de emisiones a través de los precios (como son los impuestos a las emisiones, los subsidios o los permisos) son herramientas poderosas y eficientes para regular las emisiones ${ }^{1}$.

Sin embargo, la interacción entre la políca ambiental y otras ramas de la economía crea situaciones complejas no sólo en términos del análisis económico, sino también en cuanto a sus aplicaciones prácticas. En particular, en este artículo estudiaremos la interacción entre la política medioambiental y la tecnológica. Kneese y Schultze (1975) fueron los primeros en considerar que, de entre todos los criterios disponibles para juzgar la eficiencia de los mecanismos de control de emisiones, el más importante es la medida en la cual ese mecanismo le da a las empresas fuertes incentivos a adoptar o desarrollar tecnologías menos contaminantes. Desde entonces, una gran cantidad de artículos estudia desde un punto de vista teórico y empírico, del impacto de los diferentes instrumentos de política sobre el cambio tecnológico.

Utilizando la adopción de nuevas tecnologías menos contaminantes como criterio de eficiencia, los primeros intentos para clasificar los instrumentos de política ambiental fueron hechos por Downing y White (1986), Malueg (1989) y Milliman y Prince (1989) o Junge y otros (1996). Una característica común de estos artículos teóricos es el supuesto de que el regulador actúa en forma miope, es decir, establece la política medioambiental sin tener en cuenta la posibilidad de que las empresas adopten nuevas tecnologías de reducción de emisiones. Este es el caso de la Agencia de Protección Industrial de los Estados Unidos (EPA por sus iniciales en inglés) o de algunos países europeos. La EPA se ha comprometido, por ejemplo, a un nivel constante de oferta de permisos durante los últimos 15 años. Además, en los últimos años, varios países europeos se han comprometido a niveles constantes, o incluso crecientes, de tipos impositivos sin considerar los nuevos desarrollos tecnológicos.

Sin embargo, la posibilidad de que las empresas adopten tecnologías menos contaminantes afecta a la eficiencia de una determinada política ambiental. Esta es la lógica subyacente a la Directiva 96/61 de Prevención y Control Integrado de la Contaminación (IPPC, por sus iniciales en inglés). La legislación IPPC contempla una

\footnotetext{
${ }^{1}$ Véase MULLER y MESTELMAN (1998).
} 
reducción de emisiones así como la realización de mejoras medioambientales deacuerdo con las posibilidades ofrecidas por las mejores técnicas disponibles para los distintos sectores industriales. Para ello, es necesario que la Comisión Europea organice un intercambio de información entre el regulador y las empresas involucradas sobre las Mejores Técnicas Disponibles (BAT por sus iniciales en inglés). El Instituto para el Estudio de la Prospectiva Tecnológica (IPTS por sus iniciales en inglés) organiza este intercambio de información y produce los documentos de referencia BAT que los gobiernos nacionales luego deberían tomar en consideración al decidir sobre el tipo (y nivel) de instrumentos de política ambiental a aplicar.

Requate y Unold (2003) proponen un marco de este tipo en el que el regulador anticipa la adopción de nuevas tecnologías por parte de las empresas y lo compara con una situación en la que no hay anticipación. Estos autores concluyen que cuando el regulador mueve primero y establece tanto el tipo como el nivel del instrumento de política, solo los permisos inducen un resultado eficiente.

Siguiendo el trabajo teórico de Requate y Unold $(2001,2003)$, consideramos una situación en la cual el regulador anticipa un posible cambio tecnológico al decidir sobre la política ambiental que se aplicará en una industria cuya estructura tecnológia inicial es heterogénea. Nuestro interés principal en este artículo es analizar el efecto de la heterogeneidad tecnológica de la industria en la difusión de la tecnología cuando el mecanismo de control de emisiones está basado en un sistema de permisos. A pesar de que es ampliamente reconocida la importancia de la heterogeneidad de costes a la hora de evaluar las ganancias potenciales de un mecanismo de mercado, se ha dedicado poca atención al estudio de la relación entre la heterogeneidad de la industria y el funcionamiento de los mercados. Así, nuestro trabajo se une a otra vertiente de la literatura que se concentra en el efecto de la heterogeneidad de las empresas sobre la eficiencia de las diferentes políticas de control de emisiones, como Nichols (1984) y Mendelsohn (1986). Recientemente, Newell y Stavins (2003) demuestran la relación entre el ahorro en los costes y diferentes fuentes de heterogeneidad de costes, estimando asimismo el potencial ahorro en costes derivado de utilizar un instrumento de mercado frente a un mecanismo de command and control como son los estándares uniformes. Sin embargo, ninguno de estos artículos contempla la posibilidad de que las empresas inviertan en tecnologías menos contaminantes.

Nuestros resultados experimentales pueden ayudar al regulador a la hora de decidir sobre la aplicación de diferentes programas de permisos de emisión a una determinada industria, dado que la adopción de las mejores técnicas disponibles para reducir las emisiones industriales y alcanzar el máximo beneficio medioambiental pueden conducir a un complejo equilibrio entre aplicar aquellas medidas que implican las más altas mejoras medioambientales y su coste para la industria.

El resto del artículo se estructura del siguiente modo: La Sección 2 resume los principales experimentos sobre el efecto de la inversión en tecnologías más limpias en el funcionamento del mercado de permisos de emisión. La Sección 3 introduce el marco teórico que será utilizado como referencia para analizar nuestros resulta- 
dos experimentales. La sección 4 describe el diseño experimental. Finalmente, las Sección 5 ilustra los principales resultados, mientras que la Sección 6 concluye.

\section{Literatura experimental}

Este artículo se relaciona con una gran cantidad de estudios iniciales sobre la eficiencia de los instrumentos de control de emisiones basados en mecanismos de mercado bajo diferentes supuestos. El trabajo de Plott (1983) da origen a una relativamente larga literatura experimental que estudia los mercados de permisos de emisión ${ }^{2}$. Los principales resultados de esta literatura, resumidos en Muller y Mestelman [1998], muestran que los mercados de permisos de emision funcionan. Sin embargo, son bastante sensibles a la elementos como el tipo de subasta implementada (Cason y Plott, 1996), la posibilidad de verder los permisos o de guardarlos para otro periodo (banking) (Godby y otros, 1997, o Cronshaw y Brown-Kruse, 1999), el poder de mercado (Godby, 1999), la incertidumbre (Godby y otros, 1998), etc. Sin embargo, pocos estudios experimentales han investigado el impacto de la inversión en tecnologías más limpias. Ben-David y otros (1999) analizan un mercado basado en el porgrama de permisos de emisión de la Clean Air Act y observan como el funcionamiento del mercado de permisos se ve afectado por la heterogeneidad tecnológica creada por las decisiones de inversión de las empresas. De su estudio experimental concluyen que un incremento en la heterogeneidad puede conducir a una reducción tanto en el volumen como en la eficiencia del mercado. Posteriormente, Croson y otros (2005) estudian la interacción entre banking, la inversión y la incertidumbre relativa a la asignación de los permisos. Una novedad de su trabajo es que consideran no sólo la eficiencia del mercado de permisos, sino también la eficiencia productiva. Un resultado central es que los agentes tienden a sobreinvertir. Además, a pesar de que esta sobre-inversión crea mayor eficiencia productiva, el hecho de que empresas más "sucias" inviertan y sustituyan en la producción a aquellas que eran originalmente más "limpias" hace que las inversiones sean socialmente ineficientes.

Otra serie de experimentos más recientes como Capozza (2006) o Nicklisch y Zucchini (2005) incluyen también consideraciones sobre la producción en sus diseños experimentales. Ambos artículos se centran en las diferentes estrategias individuales de las empresas y su influencia en las decisiones de inversión a fin de evaluar la eficiencia dinámica del mecanismo de permisos de emisiones en un contexto de competencia imperfecta. Los resultados de un experimento piloto confirman que el comportamiento de las empresas puede conducir a la difusión de la tecnología.

2 Véase FATÁS y ROIG [2004] para una introducción de los métodos experimentales en economía. 


\section{Marco teórico}

En esta sección describimos las principales características del modelo teórico que posteriormente utilizaremos para diseñar nuestros experimentos. El modelo que presentamos aquí es una versión discreta del introducido por Requate y Unold (2001).

Consideremos una industria compuesta por $N$ empresas, donde cada empresa $i=\{1, \ldots, N\}$ se caracteriza por la tecnología de reducción de emisiones que utiliza, que a su vez está representada por la función de costes de reducción de emisiones $C_{i}\left(e_{i}\right)$. Suponemos que en esta industria se utilizan inicialmente $K$ tecnologías diferentes. Además, existe una tecnología avanzada $I$, que la empresa puede adoptar y que es conocida por todos. Dado que las empresas con la misma tecnología elegirán el mismo nivel de emisiones en equilibrio, identificamos a cada empresa por el índice de la tecnología que emplea $k=1, \ldots, K, I$. Suponemos además que $C_{k}(e)>0$ para $e<\bar{e}_{k}$, donde $\bar{e}_{k}$ es el nivel máximo de emisiones para la tecnología $k$. La inversión en una tecnología de reducción de emisiones más avanzada, conduce tanto a un menor nivel de emisiones máximas como a un menor coste marginal de reducción de emsiones, es decir, $C_{k}^{\prime}(e)>C_{I}^{\prime}(e)>0 \mathrm{y}-C_{k}^{\prime}(e)>-C_{I}^{\prime}(e)$ para todo $e<\bar{e}_{k}$. La instalación de la nueva tecnología supone un coste fijo $F>0$, que suponemos el mismo para todas las empresas.

Además cuando el regulador fija la política óptima supone una función de daño social creciente y convexa $D(E)$ que evalúa las emisiones en términos monetarios, donde $E=\sum_{i=1}^{n} e_{i}$ representa las emisiones agregadas. En las siguientes secciones, utilizaremos una función de daño cuadrática $D(E)=E^{2} / 4$.

Denominemos $A M A C^{*}(E)$ al coste marginal de reducción de emisiones agregado $^{3}$. Así, el regulador determinará en nivel de emisiones agregado óptimo $E^{*}$ de forma que cumpla

$$
D^{\prime}(E)=A M A C^{*}\left(E^{*}\right)
$$

Nótese que el coste marginal de reducción de emisiones agregado depende del número de empresas que adopten la nueva tecnología. Suponemos que una autoridad reguladora utiliza permisos negociables para controlar las emisiones. Por lo tanto, emitirá un número de permisos negociables $L=E^{*}$ a fin de mantener el nivel de emisión agregado igual a $E^{*}$. Sea $\sigma$ el precio de mercado de los permisos, una empresa que emplea la tecnología $k$ elige un nivel de emisiones $e_{k}(\sigma)$ de forma que su coste marginal de reducción de emisiones iguale el precio de los permisos: $-C_{k}^{\prime}\left(e_{k}(s)\right)=\sigma$.

\footnotetext{
3 Aggregate Marginal Abatement Cost, en inglés.
} 
Una empresa que incialmente emplea la tecnología $k$ tiene incentivos a adoptar la nueva tecnología $I$ si:

$$
\left.\left.C_{l}\left(e_{I}(\sigma)\right)+F+\sigma e_{I}(\sigma)-\hat{e}\right]<C_{k}\left(e_{k}(\sigma)\right)+\sigma e_{k}(\sigma)-\hat{e}\right]
$$

donde $\hat{e}$ es la dotación inicial de permisos. La condición 2 dice que la inversión es beneficiosa si el coste total que incluye el coste de reducción de emisiones, los gastos por los permisos, y el coste de la inversión es menor que el coste de reducción de emisones más los gastos en permisos sin la inversión. Esta condición depende crucialmente del precio de los permisos. Incluso para empresas idénticas, es posible que, en equilibrio, algunas empresas adopten la nueva tecnología mientras que otras no lo hagan ${ }^{4}$. De hecho tanto el precio de los permisos como el número de empresas que invierten en la nueva tecnología son endógenos.

En relación al efecto de la heterogeneidad tecnológica en la industria sobre las ganacias del comercio en este mercado, parece ser consenso común que las ventajas potenciales del comercio deberían ser más altas cuanto más heterogéneo sea el mercado, dado que la distancia entre la WTA mínima y la WTP máxima será mayor ${ }^{5}$.

\section{Diseño Experimental}

A fin de implementar el marco presentado en la sección 3, definimos un conjunto de $K$ tecnologías diferentes utilizadas por las empresas y una nueva tecnología más limpia $(N T)$. La Tabla 1 ilustra las diferentes tecnologías caracterizadas por sus emisiones máximas y coste marginal de reducción de emisones, por sus iniciales en inglés $(M A C)$.

Implementamos dos tratamientos dependiendo del grando inicial de heterogeneidad tecnológica dentro de una misma industria: baja heterogeneidad y alta heterogeneidad (BH y AH, respectivamente). En la Tabla 2 presentamos la distribución inicial de las tecnologías convencionales y en la Tabla 3 la lista de los parámetros utilizados en cada tratamiento.

\footnotetext{
${ }^{4}$ Véase REQUATE y UNOLD (2003).

5 Véase BEN-DAVID y otros (1999) para una demostración teórica de la relación entre la heterogeneidad y las ganancias potenciales del comercio.
} 
TABLA 1

COSTE MARGINAL DE REDUCCIÓN DE EMISIONES (MAC) POR TIPO DE TECNOLOGÍA

\begin{tabular}{|c|c|c|c|c|c|c|c|c|c|}
\hline \multirow{2}{*}{ MAC } & \multicolumn{9}{|c|}{ Emisiones máximas $\left(\bar{e}_{k}\right)$ por tipo de tecnología } \\
\hline & T1 & $\mathbf{T} 2$ & T3 & T4 & T5 & T6 & $\mathbf{T} 7$ & T8 & NT \\
\hline 0 & 20 & 19 & 18 & 17 & 16 & 15 & 14 & 12 & 7 \\
\hline 10 & 19 & 18 & 17 & 16 & 15 & 14 & 13 & 11 & 6 \\
\hline 20 & 18 & 17 & 16 & 15 & 14 & 13 & 12 & 10 & 5 \\
\hline 30 & 17 & 16 & 15 & 14 & 13 & 12 & 11 & 9 & 4 \\
\hline 40 & 16 & 15 & 14 & 13 & 12 & 11 & 10 & 8 & 3 \\
\hline 50 & 15 & 14 & 13 & 12 & 11 & 10 & 9 & 7 & 2 \\
\hline 60 & 14 & 13 & 12 & 11 & 10 & 9 & 8 & 6 & 1 \\
\hline 70 & 13 & 12 & 11 & 10 & 9 & 8 & 7 & 5 & 0 \\
\hline 80 & 12 & 11 & 10 & 9 & 8 & 7 & 6 & 4 & 0 \\
\hline 90 & 11 & 10 & 9 & 8 & 7 & 6 & 5 & 3 & 0 \\
\hline 100 & 10 & 9 & 8 & 7 & 6 & 5 & 4 & 2 & 0 \\
\hline 110 & 9 & 8 & 7 & 6 & 5 & 4 & 3 & 1 & 0 \\
\hline 120 & 8 & 7 & 6 & 5 & 4 & 3 & 2 & 0 & 0 \\
\hline 130 & 7 & 6 & 5 & 4 & 3 & 2 & 1 & 0 & 0 \\
\hline 140 & 6 & 5 & 4 & 3 & 2 & 1 & 0 & 0 & 0 \\
\hline 150 & 5 & 4 & 3 & 2 & 1 & 0 & 0 & 0 & 0 \\
\hline 160 & 4 & 3 & 2 & 1 & 0 & 0 & 0 & 0 & 0 \\
\hline 170 & 3 & 2 & 1 & 0 & 0 & 0 & 0 & 0 & 0 \\
\hline 180 & 2 & 1 & 0 & 0 & 0 & 0 & 0 & 0 & 0 \\
\hline 190 & 1 & 0 & 0 & 0 & 0 & 0 & 0 & 0 & 0 \\
\hline 200 & 0 & 0 & 0 & 0 & 0 & 0 & 0 & 0 & 0 \\
\hline
\end{tabular}

T1, ...., T8 representan los tipos convencionales de tecnología, mientras que NT representa la nueva tecnología. 
TABLA 2

DISTRIBUCIÓN INICIAL DE LAS TECNOLOGÍAS CONVENCIONALES POR TRATAMIENTO

\begin{tabular}{|c|c|c|c|c|c|c|c|c|}
\hline \multirow{2}{*}{ Tratamiento } & \multicolumn{8}{|c|}{ Tipo de Tecnología } \\
\cline { 2 - 9 } & T1 & T2 & T3 & T4 & T5 & T6 & T7 & T8 \\
\hline BH & 2 & 2 & 2 & 2 & 2 & 2 & 2 & 0 \\
\hline AH & 4 & 0 & 3 & 0 & 4 & 0 & 3 & 4 \\
\hline
\end{tabular}

TABLA 3

VALORES DE LOS PARÁMETROS Y PREDICCIONES DE EQUILIBRIO PARA LA INVERSIÓN Y LOS PRECIOS DE LOS PERMISOS

\begin{tabular}{|c|c|c|}
\hline Parámetro & BH & AH \\
\hline$N$ & 14 & 18 \\
\hline$K$ & 7 & 5 \\
\hline$\alpha$ & 0,015 & 0,035 \\
\hline$\alpha^{*}$ & 0,141 & 0,112 \\
\hline$F$ & 580 & 580 \\
\hline$\pi_{0}$ & 1.200 & 1.200 \\
\hline$E^{*}$ & 98 & 108 \\
\hline$i^{*}$ & 6 & 7 \\
\hline$\sigma^{*}$ & 49 & 55 \\
\hline
\end{tabular}

La Tabla 3 también presenta el número óptimo de empresas que adoptan la nueva tecnología. En el caso de baja heterogeneidad estas son las empresas con las tecnologías T1, T2 y T3, mientras que en el caso de la industria altamente heterogénea, son las empresas con las tecnologías T1 y T3 las que deberían invertir. Para ambos tratamientos la Figura 1 muestra el óptimo social (caracterizado por la intersección de la curva de daño marginal y la función de coste marginal de reducción de emisiones agregado) y el equilibrio competitivo (la intersección de la función de coste marginal agregado y la oferta de permisos).

Inspirados por Newell y Stavins (2003) definimos un parámetro $\alpha$ para denotar la heterogeneidad inicial de una industria con respecto a las emisiones máximas. Este parámetro se define como el ratio entre la varianza $V\left(\bar{e}_{i}^{k}\right)$ y la media al cuadrado $\mu\left(\bar{e}_{i}^{k}\right)^{2}$ de las emisiones máximas: $\alpha=\frac{V\left(\bar{e}_{i}^{k}\right)}{\mu\left(\bar{e}_{i}^{k}\right)^{2}}$ 
En la Tabla 2 observamos que la heterogeneidad inicial $(\alpha)$ en el caso del tratamiento $\mathrm{BH}$ equivale a la mitad del valor que la del tratamiento de $\mathrm{AH}^{6}$. Sin embargo, si las empresas persiguen el óptimo en relación a sus decisiones de inversión, la heterogeneidad se incrementa $\left(\alpha^{*}\right)$ en ambos tratamientos, aumentando al mismo tiempo las ganancias potenciales del comercio. Siguiendo las prácticas reales (véase CAAA 1990 y las comercialización de las emisiones EU) consideramos un mecanismo inicial de asignación de permisos gratuito en base a las emisiones históricas (grandfathering). Por lo tanto, al comienzo de cada periodo cada empresa recibe una asignación inicial de permisos de emisión proporcional a sus emisiones máximas, presentadas en la Tabla 1. Las empresas pueden comprar y/o vender los permisos en una subasta doble ${ }^{7}$.

\section{FIGURA 1}

NIVEL DE INSTRUMENTO SOCIALMENTE OPTIMO

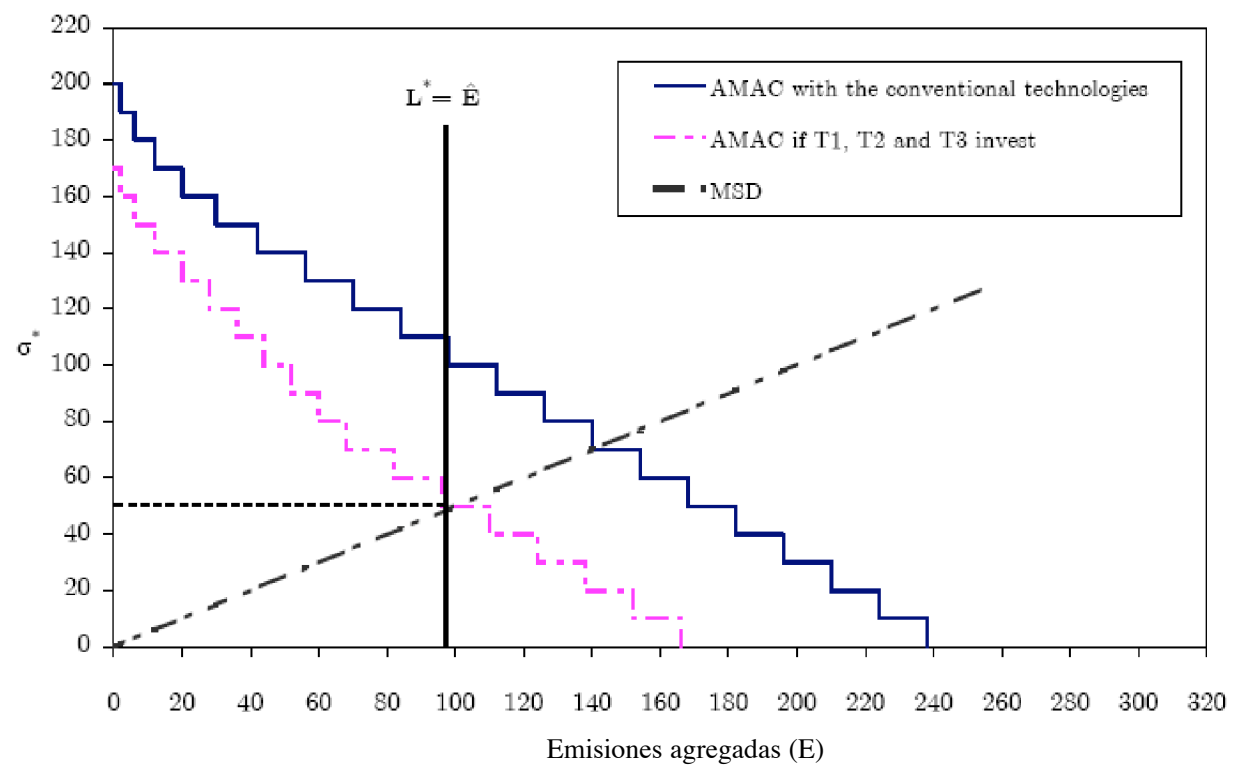

[Baja Heterogeneidad (BH)]

\footnotetext{
${ }^{6}$ Nótese que NEWELL y STAVINS (2003) reporta un valor de 0,098 para la heterogeneidad en las emisiones máximas en el caso de los óxidos de nitrógeno liberados por las Compañías de Electricidad en el este de Estados Unidos. Considerando este resultado, establecemos nuestros valores dentro de un rango realista.

7 Véase SMITH (1962) y SMITH y WILLIAMS (1992).
} 


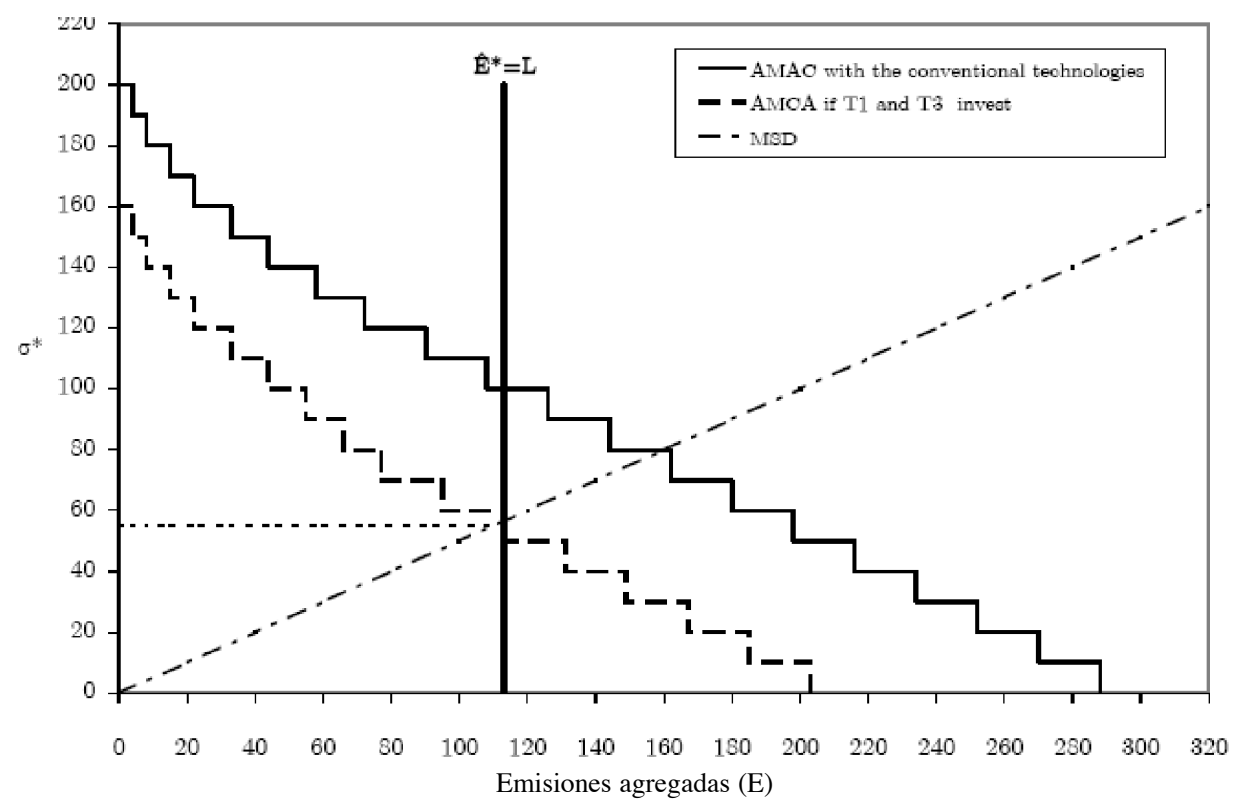

[Alta Heterogeneidad (BH)]

\subsection{Sesiones experimentales}

Las sesiones experimentales fueron realizadas en los laboratorios experimentales de la Unversidad Autónoma de Madrid (MADLEE) y de la Universidad de Kiel (KIEEL). Los sujetos son voluntarios reclutados de entre los estudiantes de (al menos) segundo año de los departamentos de Economía y Administración de Empresas de ambas universidades. Las ganancias durante los experimentos fueron denominadas en unidades monetarias experimentales (ECUs por sus iniciales en inglés) y convertidas a euros al final de cada sesión.

El experimento fue programado y conducido con el software z-Tree ${ }^{8}$. A la llegada del sujeto al laboratorio, éste fue asignado aleatoriamente a una de las terminales de ordenador. Las instrucciones ${ }^{9}$ fueron entonces distribuidas y las preguntas contestadas. Los sujetos fueron informados de que la comunicación no estaba permitida antes del final de la sesión.

Se dijo a los sujetos que cada uno de ellos representaba una empresa que utilizaba una tecnología convencional $k=1, \ldots, 8$ caracterizada por un determinado nivel de emisiones máximas y una función de costes de reducción de emisiones tal como se describió en la Tabla 1, en donde todas las empresas operan en la misma industria. Además los sujetos fueron informados de las características de la nueva tecnología (NT) disponible para todas las empresas.

8 Véase FISCHBACHER (2007).

${ }^{9}$ Las instrucciones pueden ser solicitadas a los autores. 
Cada sesión consistía en 9 (6) periodos en el caso de baja (alta) heterogeneidad. Cada período se dividía en dos etapas. En la primera etapa, se realiza la decisión de inversión. Cada sujeto decidía si conservar su tecnología convencional o adoptar la nueva pagando el correspondiente coste de inversión ${ }^{10}$. En el segundo período se abre el mercado de permisos. Los sujetos pueden comprar y/o vender permisos en una subasta doble donde cada intercambio implica un sólo permiso. Durante un período de tres minutos los sujetos fueron libres para realizar sus ofertas de compra (venta) o para comprar (vender) los permisos de emisión. Una vez que la subasta se cierra, los sujetos deberían reducir aquellas emisiones que exceden el número de permisos que poseen. Estas emisiones vienen dadas por $e_{i}=\hat{e}+\left(l_{i}^{b}-l_{i}^{s}\right)$, donde $l_{i}^{b}$ $\left(l_{i}^{s}\right)$ son el número de permisos comprados (vendidos) por la empresa $i$ en la subasta doble, y $\hat{e}_{k}$ denota la dotación inicial de la empresa $i$. La Tabla 4 describe la asignación incial de permisos de las empresas.

El beneficio de cada empresa al final del periodo se calcula del siguiente modo:

$$
\Pi_{i t}=\left\{\begin{array}{l}
\Pi^{0}-C_{k}\left(e_{k t}\right)-\sum_{j=1}^{t_{i}^{b}} \sigma_{i t}^{b}+\sum_{j=1}^{l_{j}^{*}} \sigma_{j t}^{s} \quad \text { si la empresa } i \text { usa la tecnología } k \\
\Pi^{0}-F-C_{i}\left(e_{t t}\right)-\sum_{j}^{i} \sigma_{j t}^{b}+\sum_{j=1}^{l_{i}^{s}} \sigma_{j t}^{k} \text { si la empresa } i \text { invierte }
\end{array}\right.
$$

Dado que cada transacción en la subasta doble implica el intercambio de un sólo permiso de emisión, $\sum_{j=1}^{l_{i}^{b}} \sigma_{j t}^{b}$ denota los gastos en compras de permisos y $\sum_{i=1}^{l_{i}^{s}} \sigma_{j}^{s}$ denota las ganancias de las ventas de permisos en el periodo $t$, donde $\sigma_{j t}^{b}$ y $\sigma_{j t}^{s}$ denotan los correspondientes precios de transacción.

Finalmente, los pagos finales de los sujetos se calculan sumando el beneficio obtenido en tres períodos (un período) elegidos aleatoriamente en el tratamiento $\mathrm{BH}$ $(\mathrm{AH})$.

TABLA 4

PERMISOS DISTRIBUIDOS INICIALMENTE EN FUNCIÓN DE LA TECNOLOGÍA CONVENCIONAL UTILIZADA

\begin{tabular}{|c|c|c|c|c|c|c|c|c|}
\hline $\begin{array}{c}\text { Tecnología } \\
\text { convencional }\end{array}$ & T1 & T2 & T3 & T4 & T5 & T6 & T7 & T8 \\
\hline$\hat{e}_{k}$ & 10 & 9 & 8 & 7 & 6 & 5 & 4 & 2 \\
\hline
\end{tabular}

10 Recuérdese que una vez que un sujeto ha decidido cambiar a una nueva tecnología no puede volver a la tecnología convencional. 


\section{Resultados}

\subsection{Decisiones de inversión}

La Figura 2 presenta el porcentaje de empresas de cada tipo que decide adoptar la nueva tecnología.

\section{FIGURA 2}

PORCENTAJE DE EMPRESAS QUE INVIERTEN EN LA NUEVA TECNOLOGÍA
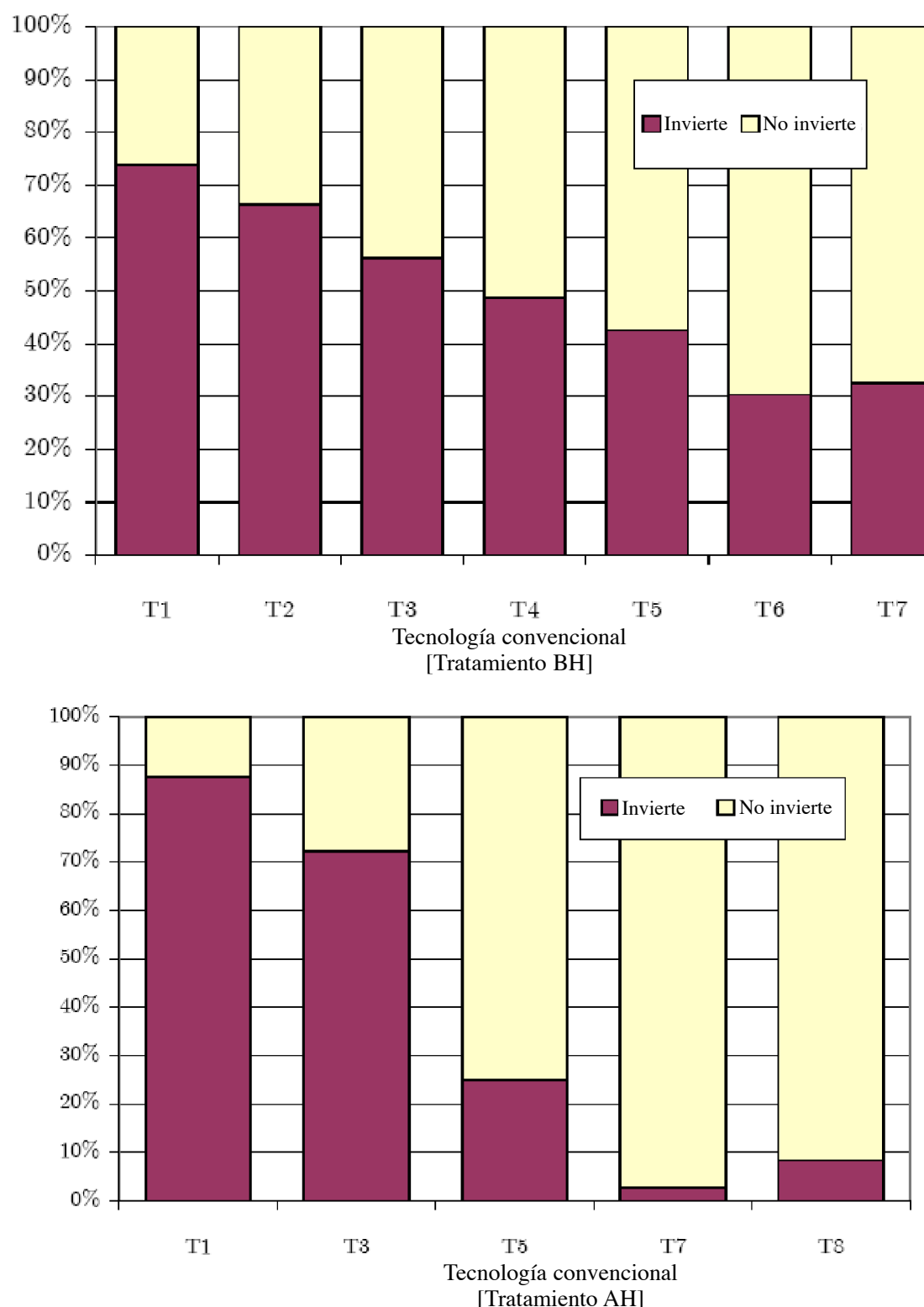
En estas figuras podemos ver que en ambos tratamientos no todas las empresas con las tecnologías más sucias (tipos 1, 2 y 3 ) invierten, a pesar de que deberían (infra-inversión), mientras que las empresas con las tecnologías convencionales más limpias (tipos 4, 5, 6, 7 y 8) lo hacen a pesar de que no deberían (sobre-inversión) ${ }^{11}$. Sin embargo, la tendencia a la sobre-inversión de las empresas con tecnologías más limpias es mayor en el caso de baja heterogeneidad ${ }^{12}$. Sin embargo, en el tratamiento con alta heterogeneidad el comportamiento en términos de inversión está más cerca de las predicciones de equilibrio descritas en la Sección 4.

Con el fin de analizar con más detalle las desviaciones del comportamiento de inversión respecto del equilibrio, construimos un índice para medir la infra(sobre)inversión de las empresas ${ }^{13}$, tomando en consideración el peso relativo de las empresas individuales en la oferta (demanda) agregada de permisos tal como se describe en el apéndice $7^{14}$.

Nuestro índice de infra-inversión se calcula como:

$$
U=\sum_{i=1}^{i^{*}} w_{i}^{s} \times n_{i}
$$

donde $n_{i}$ es el número de empresas que no invierten, mientras que deberían haberlo hecho, y $w_{i}^{s}$ es el peso de la empresa $i$ en la oferta agregada de permisos. Nótese que en equilibrio estas empresas deberían invertir, siendo los oferentes de permisos. Por lo tanto, si ellos mantienen su tecnologías convencionales (infra-inversión), la oferta de permisos será menor que la predicción de equilibrio.

En contraste, el índice de sobre-inversión se refiere al comportamiento de las restantes $N-i^{*}$ empresas:

$$
O=\sum_{i=i^{*}+1}^{N} w_{i}^{D} \times n_{i}
$$

donde $n_{i}$ es el número de empresas que invierten mientras que no deberían haberlo hecho, y $w_{i}^{D}$ representa el peso de la empresa $i$ en la demanda agregada de permisos. Dado que en equilibrio estas empresas deberían mantener sus tecnologías convencionales y comprar permisos, la demanda de los permisos se reduciría en comparación con la predicción de equilibrio.

De las ecuaciones (3) y (4) se puede ver directamente que ambos índices están acotados entre 0 y 1 . Nótese que si el índice toma un valor de 0 , esto significa que el comportamiento de las empresas frente a la inversión corresponde a la predicción

\footnotetext{
${ }^{11}$ Estos resultados están en línea con los experimentos realizados por CROSON y otros (2005) referente a la tendencia a la sobre-inversión exhibida por los agentes.

12 Un test de Wilcoxon muestra diferencias estadísticamente significativas.

${ }^{13}$ Recuérdese que la sobre(infra)-inversión se refiere al número de empresas que no invirtió (invirtió) cuando la predicción de equilibrio predice que deberían y el número de empresas que sí invirtió (no invirtió) cuando la predicción de equilibrio era que no deberían (deberían) haberlo hecho.

${ }^{14}$ Más adelante esto nos permitirá contrastar cómo los precios de los permisos reaccionan a las decisiones de inversión de las empresas.
} 
de equilibrio presentada en la Sección 4, mientras que un valor de 1 indica el comportamiento inverso.

La Tabla 5 presenta los valores de los índices de sobre e infra-inversión observados en cada uno de los tratamientos implementados.

TABLA 5

MEDIA DEL ÍNDICE DE INFRA Y SOBRE-INVERSIÓN POR PERÍODO

\begin{tabular}{|c|c|c|c|c|}
\hline \multirow{2}{*}{ Período } & \multicolumn{2}{|c|}{ BH } & \multicolumn{2}{c|}{ AH } \\
\cline { 2 - 5 } & Infra-inversión & Sobre-inversión & Infra-inversión & Sobre-inversión \\
\hline 1 & 0,50 & 0,38 & 0,26 & 0,09 \\
\hline 2 & 0,29 & 0,34 & 0,26 & 0,14 \\
\hline 3 & 0,34 & 0,50 & 0,08 & 0,23 \\
\hline 4 & 0,31 & 0,50 & 0,14 & 0,14 \\
\hline 5 & 0,34 & 0,41 & 0,28 & 0,14 \\
\hline 6 & 0,24 & 0,31 & 0,06 & 0,14 \\
\hline 7 & 0,36 & 0,28 & - & - \\
\hline 8 & 0,36 & 0,34 & - & - \\
\hline 9 & 0,42 & 0,38 & - & 0,14 \\
\hline Media & 0,35 & 0,38 & 0,18 & \\
\hline
\end{tabular}

Las desviaciones de la inversión respecto del óptimo muestran un patrón consistente. El test de Wilcoxon demuestra que dentro de un determinado tratamiento no hay diferencias estadísticamente significativas entre la sobre y la infra-inversión. Por lo tanto, concluimos que ambos tipos de desviaciones respecto del equilibrio son igualmente probables.

Cuando comparamos los dos tratamientos nuestros resultados muestran que ambos tipos de desviación respecto del comportamiento de inversión de equilibrio son significativamente más bajos en la industria heterogénea ${ }^{15}$.

Así podemos concluir que la heterogeneidad tecnológica de la industria tiene un efecto importante en el comportamiento inversor de la empresa. En una industria más heterogénea las decisiones de inversion de las empresas están más cerca del óptimo social.

Una interesante cuestión que surge en este punto se refiere a las causas de la desviación respecto del comportamiento de equilibrio esperado. Una posible explicación referente a la infra-inversión es la aplicación de una estrategía wait and see, ya

\footnotetext{
${ }^{15}$ Un test de Mann-Whitney muestra que hay una diferencia significativa entre la infra y sobreinversión entre los dos tratamientos.
} 
propuesta por Requate y Unold [2001]. Si una determinada empresa que emplea una tecnología más sucia espera que muchas otras adopten la nueva tecnología, entonces espera que el precio de los permisos caiga por debajo del precio al cual conviene invertir a aquellas empresas con tecnologías más sucias, es decir, las empresas con tecnologías $T 1, T 2$ y $T 3$ ( $T 1$ y $T 2$ ) en el tratamiento BH (AH). En consecuencia estas empresas tienen un incentivo a conservar sus tecnologías convencionales y comprar permisos adicionales en el mercado un precio menor. Por el contrario, si los precios esperados en el mercado son lo suficientemente altos, se incrementaría los incentivos a la inversión para las empresas. Nótese que los precios de los permisos en la subasta constituyen la única señal disponible para las empresas a fin de estimar las decisiones de inversión de la industria en su conjunto.

\subsection{El Mercado de los Permisos}

En esta sección nos concentramos en el mercado de permisos. Por un lado queremos saber si el mercado es capaz de reflejar la decisión de inversión de la empresa en los precios de los permisos. Por otro, dado que las empresas interactúan a través del mercado es de esperar que la evolución esperada de los precios influyan sobre la decisión de inversión de las empresas.

La Tabla 6 presenta la media de los precios y volumen observados en el mercado para cada uno de los tratamientos. Sin embargo, para evaluar la eficiencia del mercado no podemos utilizar el precio de equilibrio calculado en la Sección 4 como punto de referencia, ya que para en el cálculo del precio de equilibrio se supone que las empresas invierten de acuerdo a la predicción de equilibrio ${ }^{16}$. Por lo tanto, la Tabla 6 incluye también los precios esperados y los volúmenes dadas las decisiones de inversión de las empresas.

TABLA 6

PRECIO Y VOLUMEN MEDIO. VARIANZA ENTRE PARÉNTESIS

\begin{tabular}{|c|c|c|c|c|c|}
\hline \multirow{2}{*}{ Tratamiento } & \multicolumn{3}{|c|}{ Observado } & \multicolumn{2}{c|}{ Esperado } \\
\cline { 2 - 4 } & \multirow{2}{*}{ Precio } & \multicolumn{2}{|c|}{ Volumen } & \multirow{2}{*}{ Precio } & \multirow{2}{*}{ Volumen } \\
\cline { 3 - 4 } & & Net & Total & & \\
\hline LH & $62,73(221,7)$ & 23,87 & 38,50 & $47,61(159,0)$ & 33,70 \\
\hline HH & $64,84(92,5)$ & 30,83 & 44,16 & $55,17(76,4)$ & 35,41 \\
\hline
\end{tabular}

\footnotetext{
${ }^{16}$ Recuérdese que de la figura 1 observamos que en el tratamiento $\mathrm{BH}(\mathrm{AH})$, en equilibrio, solo aquellas empresas con una tecnología convencional de tipo 1,2, y 3 (tipo 1 y 3 ) deberían adoptar la nueva tecnología mientras que el resto de empresas deberían mantener su tecnología convencional. En este caso, el precio de equilibrio del permiso es $\sigma^{*}=49\left(\sigma^{*}=55\right)$.
} 
En el apartado anterior concluimos que la desviación de las empresas del comportamiento de inversión de equilibrio es más alto en el caso del tratamiento de $\mathrm{BH}$ comparado con el de AH. Sin embargo, dentro de un determinado tratamiento, la infra y sobre-inversión eran observadas con igual probabilidad y, por tanto, se compensan una a la otra resultando en un precio esperado de los permisos cercano al de equilibrio. Este es el caso en la Tabla 6 . Además, uno podría preguntarse si los precios observados de los permisos reflejan la conducta de inversión de las empresas.

La Tabla 6 muestra que en ambos tratamientos el mercado de los permisos sufre de una importante sobrevaloración, es decir, que el precio observado es significativamente más alto que el precio esperado teniendo en cuenta las tecnologías que utilizan las empresas. Esto es particularmente cierto en el tratamiento $\mathrm{BH}^{17}$. Además, la varianza del precio observado es más alta en el tratamiento $\mathrm{BH}$ comparada con el tratamiento AH. Estos resultados estan en línea con los hallazgos de Ben-David y otros (1999).

El mercado de los permisos sufre de sobre-precios independientemente de la heterogeneidad tecnológica de la industria.

En cuanto al volúmen incluido en la Tabla 6, distinguimos entre volúmenes netos y totales observados. Mientras que el volúmen neto se refiere a la variación en los permisos, es decir, la diferencia entre la cantidad de persmisos que la empresa posee al principio y al final de la subasta, el volúmen total indica el número total de transacciones. Nótese que la diferencia entre volúmen neto y total corresponde al comercio especulativo, dado que algunos de los sujetos compraron y vendieron permisos dentro del mismo período a fin de obtener beneficios de la dinámica de los precios. En la Tabla 6 observamos que el comercio especulativo es sustancial en ambos tratamientos y representa alrededor de un $30 \%$ del volumen total. Mientras que el volúmen neto es menor que el esperado en los dos tratamientos implementados, el volúmen total es significativamente más alto, en particular para el tratamiento AH. Por todo ello podemos conluir que las transacciones especulativas causan un exceso de volumen en el mercado de los permisos.

\subsection{Relación entre los precios y las decisiones de inversión}

Una cuestión importante en este punto se refiere al impacto de las decisiones de inversión de las empresas sobre el precio de los permisos. Se podría considerar que los permisos de emisión son comparables a activos financieros. Sin embargo, dado que constituyen insumos en el proceso de producción de las empresas, como tales,

\footnotetext{
${ }^{17}$ Nótese que, si bien los precios esperados son significativamente más altos en el tratamiento AH, cuando se comparan los precios observados, los dos tratamientos no difieren significativamente. De hecho un test de Wilcoxon muestra una diferencia estadísticamente significativa entre los precios observados y los precios esperados en los dos tratamientos. Sin embargo, un test de Mann-Whitney muestra que los precios observados no difieren significativamente entre los tratamiento, mientras que los precios esperados si lo hacen.
} 
el precio de mercado de los permisos debería reflejar cualquier cambio en el perfil teconológico de las empresas, es decir, las decisiones de inversión de las empresas en un determinado período.

Por lo tanto, en un mercado eficiente esperamos que la infra-inversión (sobreinversión) incremente (reduzca) los precios de los permisos de emisión ${ }^{18}$. A fin de contrastar esta hipótesis regresamos los precios de los permisos en función de los índices de infra(sobre)-inversión teniendo en cuenta las decisiones de inversión de las empresas.

Los resultados presentados en la Tabla 7 confirman nuestras hipótesis iniciales referente al efecto de la sobre e infra-inversión en los precios observados. Además, si consideramos el valor absoluto de los coeficientes, observamos que los precios del tratamiento BH son particularmente sensibles a la infra-inversión cuando se los compara con el efecto de la sobre-inversión. Sin embargo, en el tratamiento AH los precios de los permisos observados son sensibles tanto a la sobre, como a la infra-inversión.

TABLA 7

REGRESION MCO PARA EXPLICAR LOS PRECIOS DE LOS PERMISOS COMO FUNCIÓN DE LAS DECISIONES DE INVERSIÓN DE LAS EMPRESAS

\begin{tabular}{|c|c|c|c|c|}
\hline \multirow{2}{*}{ Variable } & \multicolumn{3}{|c|}{ BH } & \multicolumn{2}{c|}{ AH } \\
\cline { 2 - 5 } & Coef. & $p>|z|$ & Coef. & $p>|z|$ \\
\hline Constante & 61,776 & 0,000 & 70,211 & 0,000 \\
\hline Infra-inversión & 27,693 & 0,025 & 31,421 & 0,052 \\
\hline Sobre-inversión & $-23,043$ & 0,104 & $-76,696$ & 0,067 \\
\hline N. obs. & \multicolumn{3}{|c|}{36} & \multicolumn{2}{c|}{0,300} \\
\hline$R^{2}$ & \multicolumn{3}{|c|}{0,131} & \multicolumn{2}{c|}{3,350} \\
\hline F-test & \multicolumn{3}{|c|}{3,639} & \multicolumn{2}{c|}{} \\
\hline
\end{tabular}

En consecuencia podemos concluir que la rigidez del mercado para transmitir la sobre-inversión a los precios de los permisos es responsable del exceso de inversión observado. Sin embargo, este no es el caso en el tratamiento AH. En este caso, el mercado de los permisos es más eficiente transmitiendo el perfil tecnológico de la industria a los precios de los permisos, y como resultado, la disferencia entre los precios observados y esperados aunque positiva, es menor en comparación a aquella del tratamiento $\mathrm{BH}$.

\footnotetext{
${ }^{18}$ Nótese que la infra-inversión implica que aquellas empresas que en equilibrio deberían adoptar la nueva tecnología, deciden mantener su tecnología convencional, incrementando de esta manera la demanda de permisos en el mercado. Por otra parte, la sobre-inversión representa al número de empresas que adoptan una nueva tecnología a pesar de que no debería hacerlo de acuerdo a la solución óptima. Este comportamiento incrementaría la oferta de permisos de emisión y, en consecuencia, los precios esperados serían más bajos que el precio óptimo social.
} 
En la Sección 4 describimos como el modelo se implementa como un juego oneshot con tres diferentes etapas, repetidas durante un número de períodos. Por lo tanto, la única señal que los sujetos reciben sobre a la inversión de las otras empresas es el precio observado en el período anterior. La Tabla 8 presenta las estimaciones utilizando un modelo de datos de panel Probit con efectos aleatorios para explicar la probabilidad de que una empresa decida adoptar una nueva tecnología en un determinado período. Entre las variables explicativas "Tecnología Convencional en t" es una variable discreta que toma valores entre 1 y 8 e indica la tecnología convencional asignada a la empresa ${ }^{19}$ en el período $t$. "Precio en $t-1$ " indica el precio medio de los permisos en el período anterior. Utilizamos esta variable como proxy de las expectativas de las empresas sobre el comportamiento de inversión de otras empresas.

TABLA 8

MODELO PROBIT CON EFECTOS ALEATORIOS PARA EXPLICAR LAS DECISIONES DE INVERSIÓN DE LAS EMPRESAS

\begin{tabular}{|c|c|c|c|c|}
\hline \multirow{2}{*}{ Variable } & \multicolumn{2}{|c|}{ ВH } & \multicolumn{2}{|c|}{$\mathbf{A H}$} \\
\hline & Coef. & $p>|z|$ & Coef. & $p>|z|$ \\
\hline Constante & 0,3020 & 0,386 & 2,0481 & 0,093 \\
\hline $\begin{array}{l}\text { Tecnol. convencional } \\
\text { en } t\end{array}$ & $-0,2705$ & 0,000 & $-1,0672$ & 0,000 \\
\hline Precio en $t-1$ & 0,0131 & 0,006 & 0,0090 & 0,609 \\
\hline N. obs. & \multicolumn{2}{|c|}{504} & \multicolumn{2}{|c|}{181} \\
\hline N. grupos & \multicolumn{2}{|c|}{56} & \multicolumn{2}{|c|}{36} \\
\hline$\sigma_{u}$ & \multicolumn{2}{|c|}{0,8793} & \multicolumn{2}{|c|}{1,1466} \\
\hline$\rho$ & \multicolumn{2}{|c|}{0,4360} & \multicolumn{2}{|c|}{0,5679} \\
\hline Wald test & \multicolumn{2}{|c|}{62,33} & \multicolumn{2}{|c|}{36,17} \\
\hline
\end{tabular}

Los resultados presentados en la Tabla 8 muestran que la tecnología convencional es el principal determinante de las decisiones de inversión de las empresas. El coeficiente asociado a la "Tecnología Convencional en t" indica que cuando más bajas son las emisiones máximas (cuanto más limpia la tecnología es) menor es la probabilidad de inversión de una determinada empresa. Sin embargo, en el tratamiento $\mathrm{BH}$, nuestra hipótesis inicial referente al efecto indirecto de los precios de los permisos sobre la adopción de la tecnología se ve confirmada, dado que un alto precio de los permisos incrementa el incentivo de la empresa a adoptar una nueva tecnología. Sin embargo, esto no sucede en el caso en que el perfil tecnológico inicial de la industria sea más heterogéneo, es decir, en el caso del tratamiento AH.

\footnotetext{
${ }^{19}$ Nótese que cuanto mayor el valor de la variable "Tecnología Convencional en $t$ " menores son las emisiones máximas $\bar{e}_{k}$.
} 
La ineficiencia de mercado para reflejar el perfil tecnológico de la industria en los precios de los permisos induce la sobre y infra-inversión de las empresas. Como consecuencia de esto, en una industria de baja heterogeneidad un mercado de permisos ineficiente conduce a ineficiencias en la inversión de las empresas en tecnologías más limpias.

\subsection{Comparación de la Eficiencia}

En esta sección mediremos la eficiencia del mecanismo de permisos negociables para controlar las emisiones cuando las empresas pueden invertir en una tecnología más limpia. Recordemos que las ganacias esperadas del mecanismo de control de emisiones en nuestro contexto surge de dos fuentes: la inversión de la empresa en tecnologías más limpias y el mercado de permisos.

Definimos la eficiencia (relativa) como el cociente entre el bienestar observado $\left(S W_{\text {Observado }}\right)$ y el bienestar social obtenido del comportamiento de equilibrio resultante $\left(S W_{\text {Óptimo }}\right)^{20}$.

$$
E_{\text {Total }}=\frac{S W_{\text {Observado }}}{S W_{\text {Optimo }}} \times 100
$$

A pesar de que podemos calcular la eficiencia total directamente de la ecuación (5), se obtiene una mejor perspectiva de la eficiencia del mecanismo si se considera los dos componentes que multiplicativamente determinan la eficiencia total $\left(E_{\text {Total }}\right)$. El primer componente es la Eficiencia de la Inversión $\left(E_{\text {Inversión }}\right)$ y captura el grado en el cual las decisiones de inversión de la empresa se desvían del resultado de equilibrio:

$$
E_{\text {Inversión }}=\frac{S W_{\text {Esperado }}}{S W_{\text {Opimo }}} \times 100
$$

donde $S W_{\text {Esperado }}$ es el beneficio social dada la decisión de inversión de la empresa y suponiendo que el mercado de los permisos se encuentra en equilibrio dadas las decisiones de inversión de las empresas.

El segundo componente es la eficiencia del mercado $\left(E_{\text {Mercado }}\right)$ y puede interpretarse como el grado de eficiencia de mercado al reasignar los costes de reducción de emisiones entre las empresas, dadas las decisiones de inversion de la empresa:

$$
E_{\text {Mercado }}=\frac{S W_{\text {Observado }}}{S W_{\text {Esperado }}} \times 100
$$

${ }^{20}$ Definimos Beneficio Social como la suma de los beneficios de las empresas menos el daño social provocado por las emisiones. Por lo tanto, a fin de evaluar las emisiones en términos monetarios utilizamos la función de daño social $D(E)=E^{2} / 4$ introducida en la Sección 3. Donde $E=98(E=108)$ en el tratamiento $\mathrm{BH}(\mathrm{AH})$ (Véase la Tabla 3). 
De las ecuaciones (6) y (7) podemos entonces calcular la eficiencia total del siguiente modo: $E_{\text {Total }}=E_{\text {Inversión }} \times E_{\text {Mercado }}$.

De la Tabla 9 concluímos que la eficiencia total es mayor en el tratamiento AH. Cuando se comparan las tasas de eficiencia dentro de los tratamientos, un test de Wilcoxon muestra que la eficiencia de inversión es mayor que la eficiencia de mercado, especialmente para el tratamiento BH. Por ello podemos concluir que el mecanismo de permisos de emisión alcanza una mayor eficiencia total cuando se aplica a una industria (tecnológicamente) más heterogénea.

TABLA 9

RESULTADOS DE EFICIENCIA

\begin{tabular}{|c|c|c|}
\hline & $\mathbf{B H}$ & $\mathbf{A H}$ \\
\hline$S W_{\text {Observado }}$ & $7.366,78$ & $10.520,83$ \\
\hline$S W_{\text {Esperado }}$ & $8.403,17$ & $11.183,33$ \\
\hline$S W_{\text {Óptimo }}$ & 9519 & 11.870 \\
\hline$E_{\text {Total }}$ & 77,39 & 88,62 \\
\hline$E_{\text {Inversión }}$ & 88,28 & 94,21 \\
\hline$E_{\text {Mercado }}$ & 87,67 & 94,07 \\
\hline
\end{tabular}

\section{Conclusión}

Las ganancias económicas y medioambientales, en términos de beneficios sociales, esperadas de una determinada política basada en programas de permisos de emisión negociables depende de cómo se implementan. Aquí nos concentramos en un particular mecanismo que caracteriza muchas de las situaciones empíricas: un programa de permisos comercializables basado en un mercado de subastas dobles combinado con una estructura industrial que presenta heterogeneidad en las funciones de coste de reducción de emisiones entre las empresas, al tiempo que permite la posibilidad de adoptar nuevas tecnologías más limpias.

Considerando el diseño en dos etapas de nuestro experimento en relación a la decisión de inversión de las empresas y el comportamiento de mercado, nuestros resultados muestran que existe una relación directa entre la heterogeneidad tecnológica dentro de una instrustria y (i) la eficiencia de la conducta de las empresas al adoptar tecnologías más limpias y (ii) la eficiencia del mercado para capturar las ganancias del comercio por medio de la reasignación de permisos. En particular, encontramos que cuanto mayor es la heterogeneidad de la industria (medida como la distancia entre las emisiones máximas usando la tecnología convencional y las nuevas tecnologías disponibles) más fácil es para las empresas evaluar si es eficiente adoptar la nueva tecnología o no. 
Sin embargo, al estudiar la eficiencia del mercado de permisos se ve que tanto las industrias con baja como alta heterogeneidad sufren de un sustancial sobreprecio, lo que sugiere que, independientemente del grado de heterogeneidad, el mercado no refleja plenamente el comportamiento de inversión de las empresas lo cual resulta en ineficiencia. En particular, encontramos que los precios observados de los permisos no responder a la sobre-inversión, y en consecuencia, los precios de los permisos resultantes son significativamente más altos que los precios esperados. Adicionalemente, encontramos que hay exceso de volúmen tanto en las industrias de $\mathrm{BH}$ como de $\mathrm{AH}$, ya que los volúmenes totales observados son $14,2 \%$ y $24,7 \%$ más alto que sus correspondientes volúmenes esperados. Este resultado sugiere que una proporción no desdeñable de comercio especulativo esta ocrurriendo en el mercado, particularmente si lo comparamos con el volúmen neto.

Teniendo en cuenta el objetivo principal de este artículo, hemos estudiado la eficiencia global del mismo mecanismo de permisos comerciables para controlar las emisiones cuando tecnologías más limpias están disponibles, dependiendo del grado de heterogeneidad industrial relativo a las tecnologías utilizadas por las empresas. Este resultado se extiende a los componentes de inversión y eficiencia de mercado. En consecuencia, la heterogeneidad incrementa tanto la eficiencia de las decisiones de inversión de las empresas como del mercado.

\section{Referencias}

[1] BEN-DAVID, S., S. BROOKSHIRE, S. BURNESS, M. McKEE y C. SCHMIDT (1999). Heterogeneity, irreversible production choices, and effciency in emission permit market. Journal of Environmental Economics and Management, 38, pp. 176-194.

[2] I. CAPOZZA (2006). Emissions Trading and Business, chapter A Dynamic Game of Technology Diffusion under Emissions Trading: An Experiment. Physica-Verlag HD.

[2] T.N. CASON y C.R. PLOTT (1996). Epa's new emissions trading mechanism: A laboratory evaluation. Journal of Environmental Economics and Management, 30, pp. 133-160.

[3] B. CRONSHAW y J. BROWN-KRUSE (1999). Research in Experimental Economics, volume 7, chapter An experimental analysis of emission permits with banking and the Clean Air Amendments of 1990. Stamford, Conn.: JAI Press

[4] CROSON, L. GANGADHARAN, y A. FARRELL (2005). Investment decisions and emisssions reductions: Results from experiments in emissions trading. Research Paper n. 942, Department of Economics, University of Melbourne.

[5] P.B. DOWNING y L.J. WHITE (1986). Innovation in pollution control. Journal of Environmental Economics and Management, 13, pp. 18-29.

[6] E. FATÁS y J. M. ROIG (2004). Una intrducción a la economía experimental en economía. Cuadernos de Economía, 27, pp. 7-36.

[7] U. FISCHBACHER (2007). Z-tree-zurich toolbox for readymade economic experiments. Experimental Economics, 10, pp. 171-178.

[8] R. GODBY (1999). Research in Experimental Economincs, volume 7, chapter Market Power in Emission Permit Double Auctions, pp. 121-162. JAI Press. 
[9] R. GODBY, S. MESTELMAN, R. A. MULLER y D. WELLAND (1997). Emissions trading with shares and coupons when control over discharges is uncertain. Journal of Environmental Economics and Management, 32, pp. 359-381.

[10] R. GODBY, S. MESTELMAN, R. A. MULLER y D. WELLAND (1998). An experimental economic analysis of emissions trading with shares and coupons in the presence of market uncertainty. Environmetrics, 9, pp. 67-79.

[11] Ch. JUNG, K. KRUTILLA y R. BOYD (1996). Incentives for advanced pollution abatement technology at the industry level: An evaluation of policy alternatives. Journal of Environmental Economics and Management, 30, pp. 95-111.

[12] A. KNEESE y C. SCHULTZE (1975). Pollution, Prices and Public Policy. Washington, D.C.: Brookings Institution.

[13] D.A. MALUEG (1989). Emission credit trading and the incentive to adopt new policy abatement technology. Journal of Environmental Economics and Management, 18, pp. 52-57.

[14] R. MENDELSOHN (1986). Regulating heterogeneous emissions. Journal of Environmental Economics and Management, 13, pp. 301-312.

[15] S.R. MILLIMAN y R. PRINCE (1989). Firm incentives to promote technological change in pollution control. Journal of Environmental Economics and Management, 17, pp. 247-265.

[16] R. A. MULLER y S. MESTELMAN (1998). What have we learned from emissions trading experiments? Managerial and Decision Economics, 19, pp. 225-238.

[17] R. G. NEWELL y R. N. STAVINS (2003). Cost heterogeneity and the potential savings from market-based policies. Journal Regulatory Economics, 23, 43-59.

[18] A. L. NICHOLS (1984). Targeting Economic Incentives for Environmental Protection. Cambridge: MIT Press.

[19] A. NICKLISCH y L. ZUCCHINI. Dynamic efficiency of emission trading markets: An experimental study. Discussion Papers on Strategic Interaction 2005-07, Max Planck Institute of Economics, Strategic Interaction Group, 2005. URL http://ideas.repec.org/ p/esi/discus/2005-07.html.

[20] C. A. PLOTT (1983). Externalities and corrective policies in experimental markets. The Economic Journal, 93, pp. 106-127.

[21] T. REQUATE y W. UNOLD (2001). On the incentives created by policy instruments to adopt advanced abatement technology if firms are asymmetric. Journal of Institutional and Theoretical Economics, 157, pp. 536-554.

[22] T. REQUATE y W. UNOLD (2003). Environmental policy incentives to adopt advanced abatement technology: Will the true ranking please stand up? European Economic Review, 47, pp. 125-146.

[23] V. L. SMITH (1962). An experimental study of competitive market behavior. Journal of Political Economy, 70, pp. 111-137.

[24] V. L. SMITH y A. W. WILLIAMS (1992). Experimental market economics. Scientific American, 267, pp. 116-122. 


\section{APÉNDICE \\ PESO INDIVIDUAL DE LA EMPRESA EN EL ÍNDICE DE INFRA Y SOBRE-INVERSIÓN}

En lo que sigue describimos como se calculan los pesos incluidos en los índices de infra y sobre- inversión. Las expresiones para las funciones de oferta $\left(L_{S}\right)$ y demanda agregada $\left(L_{D}\right)$ dado el precio de los permisos, $\sigma$ son $L_{S}=L_{S}=\sum_{i=1}^{i^{*}} L_{S i} \mathrm{y}$, $L_{D}=\sum_{i=i^{*}+1}^{N} L_{D i}$ respectivamente. Nótese que $i^{*}$ indica el número de empresas que en equilibrio se espera que adopten la nueva tecnología, es decir las empresas que utilizan las tecnologías convencionales T1, T2 y T3 (T1 y T3) en el tratamiento $\mathrm{BH}(\mathrm{AH})$.

La oferta individual depende de la tecnología convencional utilizada por cada empresa:

$$
L_{S i}=\left(\hat{e}_{i}-\bar{e}^{I}\right)+0.10 \sigma
$$

donde $\bar{e}^{I}=7$ y $\hat{e}_{i}$ son el número de permisos distribuídos (grandfathering). Por lo tanto, definimos el peso individual de la empresa en la oferta agregada de permisos como:

$$
w_{i}^{S}=\frac{L_{S i}}{L_{S}} .
$$

En relación con la demanda de permisos, nótese que la expresión de la demanda individual de permisos es:

$$
L_{D i}=\left(\bar{e}_{k}-\hat{e}_{i}\right)-0.1 \sigma=10-0.1 \sigma
$$

Entonces, definimos el peso individual de la empresa en la demanda agregada de permisos como:

$$
w_{i}^{D}=\frac{L_{D i}}{L_{D}}
$$

La Tabla 10 presenta el peso individual de las empresas utilizando la tecnología $k$ en la oferta $\left(w_{i}^{S}\right)$ y demanda $\left(w_{i}^{D}\right)$ agregada de permisos para los tratamiento $\mathrm{BH}(\mathrm{AH})^{21}$.

\footnotetext{
${ }^{21}$ Nótese que al calcular los pesos utilizamos las funciones de oferta y demanda evaluadas al precio de equilibrio $\sigma^{*}$. Véase la Tabla 4.
} 
TABLA 10

PESOS INDIVIDUALES DE LAS EMPRESAS

POR TECNOLOGÍA Y TRATAMIENTO

\begin{tabular}{|c|c|c|c|c|c|c|c|c|}
\hline Tratamiento & $\boldsymbol{w}_{\mathbf{1}}^{\boldsymbol{S}}$ & $\boldsymbol{w}_{\mathbf{2}}^{\boldsymbol{S}}$ & $\boldsymbol{w}_{\mathbf{3}}^{\boldsymbol{S}}$ & $\boldsymbol{w}_{\mathbf{4}}^{\boldsymbol{S}}$ & $\boldsymbol{w}_{\mathbf{5}}^{\boldsymbol{S}}$ & $\boldsymbol{w}_{\mathbf{6}}^{\boldsymbol{S}}$ & $\boldsymbol{w}_{\mathbf{7}}^{\boldsymbol{S}}$ & $\boldsymbol{w}_{\mathbf{8}}^{\boldsymbol{S}}$ \\
\hline $\mathrm{BH}$ & 0,191 & 0,165 & 0,143 & 0,125 & 0,125 & 0,125 & 0,125 & - \\
\hline $\mathrm{AH}$ & 0,160 & - & 0,120 & - & 0,09 & - & 0,09 & 0,09 \\
\hline
\end{tabular}

